\title{
CONSTRUCTION OF NORMAL NUMBERS BY CLASSIFIED PRIME DIVISORS OF INTEGERS II
}

\author{
Jean-Marie De Koninck, ImRe Kátai
}

\begin{abstract}
Given an integer $q \geqslant 2$, a $q$-normal number is an irrational number $\eta$ such that any preassigned sequence of $k$ digits occurs in the $q$-ary expansion of $\eta$ at the expected frequency, namely $1 / q^{k}$. In a series of recent papers, using the complexity of the multiplicative structure of integers along with a method we developed in 1995 regarding the distribution of subsets of primes in the prime factorization of integers, we initiated new methods allowing for the creation of large families of normal numbers. Here, we further expand on this initiative.
\end{abstract}

Keywords: normal numbers, primes, arithmetic function.

\section{Introduction}

Given an integer $q \geqslant 2$, a $q$-normal number, or simply a normal number, is an irrational number whose $q$-ary expansion is such that any preassigned sequence, of length $k \geqslant 1$, of base $q$ digits from this expansion, occurs at the expected frequency, namely $1 / q^{k}$.

Let $A_{q}=\{0,1, \ldots, q-1\}$ be the set of digits in base $q$. An expression of the form $i_{1} i_{2} \ldots i_{k}$, where each $i_{j} \in A_{q}$, is said to be a word of length $k$. Given a word $\alpha$, we shall write $\lambda(\alpha)=t$ to indicate that $\alpha$ is a word of length $t$. We shall also use the symbol $\Lambda$ to denote the empty word and write $\lambda(\Lambda)=0$. Also, we let $A_{q}^{k}$ stand for the set of all words of length $k$ and $A_{q}^{*}$ stand for the set of all the words regardless of their length.

In 1995 (see [1]), we introduced the notion of a disjoint classification of primes, that is a collection of $q+1$ disjoint sets of primes $\mathcal{R}, \wp_{0}, \wp_{1}, \ldots, \wp_{q-1}$, whose union is $\wp$, the set of all primes, where $\mathcal{R}$ is a finite set (perhaps empty) and where the other $q$ sets are of positive densities $\delta_{0}, \delta_{1}, \ldots, \delta_{q-1}$ (with clearly $\sum_{i=0}^{q-1} \delta_{i}=1$ ). We then introduced the function $H: \mathbb{N} \rightarrow A_{q}^{*}$ defined by $H(n)=H\left(p_{1}^{a_{1}} \cdots p_{r}^{a_{r}}\right)=\ell_{1} \ldots \ell_{r}$,

The first author was partially supported by a grant from NSERC. The second author was partially supported by a grant from the European Union and the European Social Fund TÁMOP 4.2.1/B-09/1/KMR-2010-0003.

2010 Mathematics Subject Classification: primary: 11K16; secondary: 11N37, 11A41 
where each $\ell_{j}$ is such that $p_{j} \in \wp_{\ell_{j}}$, and investigated the size of the set of positive integers $n \leqslant x$ for which $H(n)=\alpha$ for a given word $\alpha \in A_{q}^{k}$. More precisely, letting $\omega(n)$ stand for the number of distinct prime factors of $n$, and letting $P(n)$ and $p(n)$ stand respectively for the largest and smallest prime factor of $n$, writing $\pi(B)$ for the number of primes belonging to the set $B$ and writing $x_{1}=\log x$, $x_{2}=\log x_{1}$ and so on, we proved the following result.

Theorem A. Let $\mathcal{R}, \wp_{0}, \wp_{1}, \ldots, \wp_{q-1}$ be a disjoint classification of primes such that

$$
\pi\left([u, u+v] \cap \wp_{i}\right)=\delta_{i} \pi([u, u+v])+O\left(\frac{u}{\log ^{c_{1}} u}\right)
$$

holds uniformly for $2 \leqslant v \leqslant u, i=0,1, \ldots, q-1$, where $c_{1} \geqslant 5$ is a constant, $\delta_{0}, \delta_{1}, \ldots, \delta_{q-1}$ are positive constants such that $\sum_{i=0}^{q-1} \delta_{i}=1$. Let $\lim _{x \rightarrow \infty} w_{x}=$ $+\infty, w_{x}=O\left(x_{3}\right), \sqrt{x} \leqslant Y \leqslant x$ and $1 \leqslant k \leqslant c_{2} x_{2}$, where $c_{2}$ is an arbitrary constant. Let $A$ be a positive integer such that $A \leqslant x_{2}$ and $P(A) \leqslant w_{x}$. Then,

$$
\begin{aligned}
\#\left\{n=A n_{1} \leqslant Y\right. & \left.: p\left(n_{1}\right)>w_{x}, \omega\left(n_{1}\right)=k, H\left(n_{1}\right)=i_{1} \ldots i_{k}\right\} \\
& =(1+o(1)) \delta_{i_{1}} \cdots \delta_{i_{k}} \frac{Y}{A \log Y} t_{k}(Y) \varphi_{w_{x}}\left(\frac{k-1}{x_{2}}\right) F\left(\frac{k-1}{x_{2}}\right),
\end{aligned}
$$

where $t_{k}(x)=\frac{x_{2}^{k-1}}{(k-1) !}$

$$
\varphi_{w}(z):=\prod_{p \leqslant w}\left(1+\frac{z}{p}\right)^{-1} \quad \text { and } \quad F(z):=\frac{1}{\Gamma(z+1)} \prod_{p}\left(1+\frac{z}{p}\right)\left(1-\frac{1}{p}\right)^{z} \text {. }
$$

In 2011 (see [2]), we used Theorem A to construct large families of normal numbers, namely by establishing the following result.

Theorem B. Let $q \geqslant 2$ be an integer and let $\mathcal{R}, \wp_{0}, \wp_{1}, \ldots, \wp_{q-1}$ be a disjoint classification of primes. Assume that, for a certain constant $c_{1} \geqslant 5$,

$$
\pi\left([u, u+v] \cap \wp_{i}\right)=\frac{1}{q} \pi([u, u+v])+O\left(\frac{u}{\log ^{c_{1}} u}\right)
$$

uniformly for $2 \leqslant v \leqslant u, i=0,1, \ldots, q-1$, as $u \rightarrow \infty$. Furthermore, let $H: \wp \rightarrow$ $A_{q}^{*}$ be defined by

$$
H(p)= \begin{cases}\Lambda & \text { if } p \in \mathcal{R}, \\ \ell & \text { if } p \in \wp_{\ell} \text { for some } \ell \in A_{q}\end{cases}
$$

and further let $T: \mathbb{N} \rightarrow A_{q}^{*}$ be defined by $T(1)=\Lambda$ and for $n \geqslant 2$ by

$$
T(n)=T\left(p_{1}^{a_{1}} \cdots p_{r}^{a_{r}}\right)=H\left(p_{1}\right) \ldots H\left(p_{r}\right) .
$$

Then, the number $0 . T(1) T(2) T(3) T(4) \ldots$ is a $q$-normal number. 
As one will notice, Theorem B does not use the full power of Theorem A. Indeed, it is clear that condition (1.2) is much more restrictive than condition (1.1) since it does not allow for subsets of primes $\wp_{j}$ of distinct densities. In this paper, we will first weaken condition (1.2) to allow for the construction of even larger families of normal numbers. Then, we will extend our method in order to construct normal numbers using the sequence of shifted primes, and thereafter using the sequence $n^{2}+1, n=1,2, \ldots$

Finally, let us mention some notations we shall be using. As usual, $\varphi$ will stand for the Euler function and $\operatorname{li}(x)$ for the logarithmic integral, that is $\operatorname{li}(x)=\int_{0}^{x} \frac{d t}{\log t}$. Throughout this text, the letters $p, p_{1}, p_{2}, \ldots, q_{1}, q_{2}, \ldots, \pi_{0}, \pi_{1}, \pi_{2}, \ldots$ will always denote primes, while the letter $c$ will stand for a positive constant, but not necessarily the same at each occurrence.

\section{Main results}

Theorem 1. Assume that $\mathcal{R}, \wp_{0}, \ldots, \wp_{q-1}$ are disjoint sets of primes, whose union is $\wp$, and assume that there exists a positive number $\delta<1$ and a real number $c_{1} \geqslant 5$ such that

$$
\pi\left([u, u+v] \cap \wp_{i}\right)=\delta \pi([u, u+v])+O\left(\frac{u}{\log ^{c_{1}} u}\right)
$$

holds uniformly for $2 \leqslant v \leqslant u, i=0,1, \ldots, q-1$, and similarly

$$
\pi([u, u+v] \cap \mathcal{R})=(1-q \delta) \pi([u, u+v])+O\left(\frac{u}{\log ^{c_{1}} u}\right) .
$$

Let $H$ and $T$ be defined as in (1.3) and (1.4). Then,

$$
\xi=0 . T(1) T(2) T(3) \ldots
$$

is a q-normal number.

\section{Examples}

1. Let $\wp_{0}=\{p: p \equiv 1(\bmod 8)\}, \wp_{1}=\{p: p \equiv 7(\bmod 8)\}$ and $\mathcal{R}=\{2\} \cup\{p:$ $p \equiv 3,5(\bmod 8)\}$. With $H, T$ and $\xi$ as in the statement of Theorem 1 , we may conclude that the number $\xi$ is a binary normal number.

2. Let $P(x)=e_{k} x^{k}+\cdots+e_{1} x \in \mathbb{R}[x]$ be a polynomial with at least one irrational coefficient. Let $I_{0}$ and $I_{1}$ be two disjoint intervals in $[0,1)$ of equal length. Consider the set of primes $\wp_{0}=\left\{p:\{P(p)\} \in I_{0}\right\}, \wp_{1}=\left\{p:\{P(p)\} \in I_{1}\right\}$ and $\mathcal{R}=\wp \backslash\left(\wp_{0} \cup \wp_{1}\right)$. (Here, $\{P(p)\}$ stands for the fractional part of $P(p)$.) With $H, T$ and $\xi$ as in Theorem 1, we may conclude that $\xi$ is a binary normal number.

3. It is well known that, given a prime $p \equiv 1(\bmod 4)$, there exists a prime $\rho \in \mathbb{Z}[i]$ (the set of Gaussian integers) such that $\frac{\arg \rho}{\pi / 2} \in[0,1)$ and $p=\rho \cdot \bar{\rho}$. 
So, let the subsets of primes $\wp_{0}, \ldots, \wp_{q-1}$ be defined in such a way that $p \in \wp_{j}$ if the corresponding Gaussian prime $\rho$ satisfies

$$
\frac{\arg \rho}{\pi / 2} \in\left[\frac{j}{q}, \frac{j+1}{q}\right) \quad(j=0,1, \ldots, q-1)
$$

and let $\mathcal{R}=\{2\} \cup\{p: p \equiv 3(\bmod 4)\}$. Then, letting $H, T$ and $\xi$ be defined as in Theorem 1, we get that $\xi$ is a normal number in base $q$.

Theorem 2. Let $\mathcal{R}, \wp_{0}, \ldots, \wp_{q-1}, H$ and $T$ be as in the statement of Theorem 1. Then the number

$$
\eta=0 . T(1) T(2) T(4) T(6) T(10) \ldots T(p-1) \ldots,
$$

where $p$ runs through the sequence of primes, is a q-normal number.

Theorem 3. Let $f: \mathbb{N} \rightarrow \mathbb{N}$ be defined by $f(n)=n^{2}+1$. Consider the subset of primes $\widetilde{\wp}:=\{p \in \wp: p \equiv 1(\bmod 4)\}$. Assume that the sets $\wp_{0}, \wp_{1}, \ldots, \wp_{q-1} \subseteq \widetilde{\wp}$ satisfy (2.1) and let

$$
\mathcal{R}=\wp \backslash\left(\bigcup_{j=0}^{q-1} \wp_{j}\right) .
$$

Let also $H$ and $T$ be defined as in (1.3) and (1.4). Then

$$
\tau=0 . T(f(1)) T(f(2)) T(f(3)) T(f(4)) \ldots
$$

is a q-normal number.

\section{Preliminary results}

Lemma 1. Let $f(n)$ be a real valued non negative arithmetic function. Let $a_{n}$, $n=1, \ldots, N$, be a sequence of integers. Let $r$ be a positive real number, and let $p_{1}<p_{2}<\cdots<p_{s} \leqslant r$ be prime numbers. Set $Q=p_{1} \cdots p_{s}$. If $d \mid Q$, then let

$$
\sum_{\substack{n=1 \\ a_{n} \equiv 0}}^{N} f(n)=\kappa(d) X+R(N, d),
$$

where $X$ and $R$ are real numbers, $X \geqslant 0$, and $\kappa\left(d_{1} d_{2}\right)=\kappa\left(d_{1}\right) \kappa\left(d_{2}\right)$ whenever $d_{1}$ and $d_{2}$ are co-prime divisors of $Q$.

Assume that for each prime $p, 0 \leqslant \kappa(p)<1$. Setting

$$
I(N, Q):=\sum_{\substack{n=1 \\\left(a_{n}, Q\right)=1}}^{N} f(n),
$$


then the estimate

$$
I(N, Q)=\left\{1+2 \theta_{1} H\right\} X \prod_{p \mid Q}(1-\kappa(p))+2 \theta_{2} \sum_{\substack{d \mid Q \\ d \leqslant z^{3}}} 3^{\omega(d)}|R(N, d)|
$$

holds uniformly for $r \geqslant 2, \max (\log r, S) \leqslant \frac{1}{8} \log z$, where $\left|\theta_{1}\right| \leqslant 1,\left|\theta_{2}\right| \leqslant 1$, and

$$
H=\exp \left(-\frac{\log z}{\log r}\left\{\log \left(\frac{\log z}{S}\right)-\log \log \left(\frac{\log z}{S}\right)-\frac{2 S}{\log z}\right\}\right)
$$

and

$$
S=\sum_{p \mid Q} \frac{\kappa(p)}{1-\kappa(p)} \log p .
$$

When these conditions are satisfied, there exists an absolute positive constant $c$ such that $2 H \leqslant c<1$.

Proof. This result is Lemma 2.1 in the book of Elliott [3].

Lemma 2 (Brun-Titchmarsh inequality). There exists a positive constant $c$ such that

$$
\pi(x ; k, \ell)<c \frac{x}{\varphi(k) \log (x / k)} \quad \text { for all } k<x .
$$

Proof. For a proof, see the book of Halberstam and Richert [4].

Lemma 3 (Bombieri-Vinogradov theorem). Given any fixed number $C>0$, there exists a number $B=B(C)>0$ such that

$$
\sum_{k \leqslant \sqrt{x} /\left(\log ^{B} x\right)} \max _{(k, \ell)=1} \max _{y \leqslant x}\left|\pi(y ; k, \ell)-\frac{l i(y)}{\varphi(k)}\right|=O\left(\frac{x}{\log ^{C} x}\right) .
$$

Proof. For a proof, see the book of Iwaniec and Kowalski [5].

For the statement of the next results, we shall need the following notations.

Let $Z_{x}$ be a function tending to infinity but with the condition $\frac{\log Z_{x}}{\log x} \rightarrow 0$ as $x \rightarrow \infty$. Furthermore, let $K_{x} \rightarrow \infty$ as $x \rightarrow \infty$, but also satisfying $\frac{K_{x} \log Z_{x}}{\log x} \rightarrow 0$ as $x \rightarrow \infty$.

Let $Q=\prod_{p \leqslant Z_{x}} p$. Given an integer $m \geqslant 2$ such that $P(m) \leqslant Z_{x}$, we set

$$
\mathcal{A}(x \mid m)=\#\left\{p \leqslant x: p \equiv 1(\bmod m), \operatorname{gcd}\left(\frac{p-1}{m}, Q\right)=1\right\} .
$$

Further set $\nu(Q)=\prod_{\substack{p \mid Q \\ p>2}}\left(1-\frac{1}{p-1}\right)$ 
We now introduce the strongly multiplicative function $\kappa(n)$ defined on primes $p$ by

$$
\kappa(p)= \begin{cases}1 & \text { if } p=2 \\ \frac{p-1}{p-2} & \text { if } p>2\end{cases}
$$

Lemma 4. Let $Z_{x}$ and $K_{x}$ be defined by $\log Z_{x}=(\log x) / x_{2}^{2}$ and $K_{x}=B x_{2}$, where $B$ is a large constant. Then, given any arbitrarily large constant $C$,

$$
\sum_{\substack{m \leqslant Z_{x} K_{x} \\ P(m) \leqslant Z_{x}}}\left|\mathcal{A}(x \mid m)-\frac{\nu(Q) \kappa(m)}{m} l i(x)\right| \ll \frac{x}{\log ^{C} x} .
$$

Proof. For now, we fix an integer $m \leqslant Z_{x}^{K_{x}}$ such that $P(m) \leqslant Z_{x}$. We plan to use Lemma 1. For this, we set $r=\pi\left(Z_{x}\right)$ and we let $q_{1}<\cdots<q_{T}$ be the sequence of those primes $q_{j} \leqslant x$ satisfying $q_{j}-1 \equiv 0(\bmod m)$ for $j=1, \ldots, T$ (so that $T=\pi(x ; m, 1)$ ); and also we let $a_{n}=\left(q_{n}-1\right) / m$ for $n=1,2, \ldots, T$ and set $f(n)=1$. Now, define $R(m, d)$ implicitly by

$$
\pi(x ; d m, 1)=\sum_{\substack{p \leqslant x \\ \frac{p-1}{m} \equiv 0 \quad(\bmod d)}} 1=\eta(d) \pi(x ; m, 1)+R(m, d),
$$

where $\eta(d)$ is the strongly multiplicative function defined on primes $p$ by

$$
\eta(p)= \begin{cases}\frac{1}{p} & \text { if } p \mid m, \\ \frac{1}{p-1} & \text { if }(p, m)=1 .\end{cases}
$$

Hence, as a consequence of Lemma 1, we obtain

$$
\mathcal{A}(x \mid m)=\left\{1+2 \theta_{1} H\right\} \pi(x ; m, 1) \prod_{p \mid Q}(1-\eta(p))+2 \theta_{2} \sum_{\substack{d \mid Q \\ d \leqslant z^{3}}} 3^{\omega(d)}|R(m, d)| .
$$

Now, since

$$
S=\sum_{\substack{p \mid Q \\ p>2}} \frac{\log p}{p-2}=(1+o(1)) \log Z_{x} \quad(x \rightarrow \infty)
$$

and

$$
r=\pi\left(Z_{x}\right) \quad \text { and } \quad \log r=\log Z_{x}+O(\log \log x)
$$

and since

$$
\log z=K_{x} \log Z_{x}, \quad \frac{\log z}{\log r} \sim K_{x}, \quad \log \left(\frac{\log z}{S}\right)=\log K_{x} \quad(x \rightarrow \infty),
$$

we have, for $x$ large,

$$
H=\exp \left\{-K_{x}\left(\log K_{x}-\log \log K_{x}-z / K_{x}\right)\right\} \leqslant \exp \left\{-\frac{K_{x}}{2} \log K_{x}\right\} .
$$


Hence, it follows from (3.3) that

$$
\begin{aligned}
& \left|\mathcal{A}(x \mid m)-\pi(x ; m, 1) \frac{\varphi(m)}{m} \kappa(m) \nu(Q)\right| \\
& \leqslant 2 H \pi(x ; m, 1) \nu(Q) \kappa(m)+2 \sum_{\substack{d \mid Q \\
d \leqslant z^{3}}} 3^{\omega(d)}|R(m, d)|,
\end{aligned}
$$

where $R(m, d)$ satisfies, in light of (3.2),

$$
|R(m, d)| \leqslant E(d m)+\frac{E(m)}{\varphi(d)}
$$

where

$$
E(r):=\left|\pi(x ; r, 1)-\frac{\operatorname{li}(x)}{\varphi(r)}\right| .
$$

Using (3.5), we have that

$$
\begin{aligned}
\sum_{\substack{d \mid Q \\
d \leqslant z^{3}}} 3^{\omega(d)}|R(m, d)| & \leqslant \sum_{\substack{d \mid Q \\
d \leqslant z^{3}}} 3^{\omega(d)}\left(E(d m)+\frac{E(m)}{\varphi(d)}\right) \\
& =\sum_{\substack{d \mid Q \\
d \leqslant z^{3}}} 3^{\omega(d)} E(d m)+\sum_{\substack{d \mid Q \\
d \leqslant z^{3}}} 3^{\omega(d)} \frac{E(m)}{\varphi(d)} \\
& =\sum_{1}+\sum_{2},
\end{aligned}
$$

say. Now, on the one hand,

$$
\sum_{1}=\sum_{k \leqslant z^{4}} E(k) \prod_{p \mid k}(1+3)=\sum_{k \leqslant z^{4}} E(k) 2^{2 \omega(k)} .
$$

On the other hand, we have

$$
\sum_{2} \leqslant E(m) \sum_{d \mid Q} \frac{3^{\omega(d)}}{\varphi(d)} \leqslant E(m) \prod_{p \mid Q}\left(1+\frac{3}{p-1}\right) \leqslant c E(m)\left(\log Z_{x}\right)^{3} .
$$

Thus, using (3.7) and (3.8) in (3.6), we obtain that

$$
\begin{aligned}
\sum_{\substack{d \mid Q \\
d \leqslant z^{3}}} 3^{\omega(d)}|R(m, d)| & \leqslant c\left(\log Z_{x}\right)^{3} E(m)+\sum_{k \leqslant z^{4}} E(k) 2^{2 \omega(k)} \\
& =T_{1}+T_{2}
\end{aligned}
$$

say. Now, because of Lemma 3, we have that, given any fixed constant $C$,

$$
T_{1} \ll \frac{x}{\log ^{C} x} .
$$


On the other hand, observe that since $a \leqslant b+\frac{1}{b} a^{2}$ for all $a, b \in \mathbb{R}^{+}$, we have

$$
T_{2} \leqslant 2^{2 B x_{2}} \sum_{k \leqslant z^{4}} E(k)+2^{-2 B x_{2}} \sum_{k \leqslant z^{4}} E(k) 2^{4 \omega(k)}=U_{1}+U_{2},
$$

say. Using Lemmas 3 and 2 in order to estimate $U_{1}$ and $U_{2}$, respectively, it follows that (3.11) can be replaced by

$$
T_{2} \leqslant \frac{x}{(\log x)^{\left(A^{\prime}\right)(2 B \log 2)}}+\frac{x}{\log x}(\log x)^{-2 B \log 2} \sum_{k \leqslant z^{4}} \frac{2^{4 \omega(k)}}{\varphi(k)},
$$

where $B$ and $A^{\prime}$ are arbitrary positive constants. Hence, by an appropriate choice of $B$ and $A^{\prime}$, it follows from (3.12) that

$$
T_{2} \ll \frac{x}{\log ^{C} x} .
$$

Then, using (3.10) and (3.13) in (3.9), placing the result in (3.4) and then summing the first term on the right hand side of (3.4) over $m$, we obtain from Lemma 2 that it is $\ll x /\left(\log ^{C} x\right)$, thus completing the proof of Lemma 4 .

Lemma 5. Given positive integers $k$ and $A$, set

$$
B_{k}(x, A)=\sum_{\substack{m_{1} \leqslant Z_{x}^{K} \\ \omega\left(m_{1}\right)=k \\ \text { and } \\ p\left(m_{1}\right)>w_{x}, P\left(m_{1}\right) \leqslant z_{x}}} \mathcal{A}\left(x \mid A m_{1}\right) .
$$

Let $\wp_{0}, \ldots, \wp_{q-1}$ be a disjoint classification of primes with corresponding densities $\delta_{0}, \ldots, \delta_{q-1}$. Then, given an arbitrary constant $C>0$,

$$
\sum_{\substack{A \leqslant w_{x} \\ P(A) \leqslant w_{x}}} \sum_{k \leqslant B x_{2}} \sum_{\substack{x \\ i_{1} \ldots i_{k} \in A_{q}^{k}}}\left|\sum_{\substack{\left.m_{1} \leqslant z_{x}^{K} \\ H\left(m_{1}\right)=i_{1} \ldots i_{k} \\ p\left(m_{1}\right)>w_{x}, m_{1}\right) \leqslant z_{x}}} \mathcal{A}\left(x \mid A m_{1}\right)-\delta_{i_{1}} \cdots \delta_{i_{k}} B_{k}(x, A)\right|
$$

Moreover,

$$
\begin{aligned}
& \sum_{\substack{A \leqslant w_{x}^{w} w_{x} \\
P(A) \leqslant w_{x}}} \sum_{k \leqslant B x_{2}}\left|B_{k}(x, A)-\nu(Q) \operatorname{li}(x) \frac{\kappa(A)}{A} \sum_{\substack{m_{1} \leqslant Z_{x}^{K} \\
\omega\left(m_{1}\right)=k \\
\text { a } \\
p\left(m_{1}\right)>w_{x}, P\left(m_{1}\right) \leqslant Z_{x}}} \frac{\kappa\left(m_{1}\right)}{m_{1}}\right| \\
& \ll \frac{x}{\log ^{C} x} .
\end{aligned}
$$


Proof. The result is a direct consequence of Theorem A and Lemma 4.

Lemma 6. Given positive integers $h \geqslant 2 k$,

$$
\sum_{\alpha \in A_{q}^{h}}\left(F_{\beta}(\alpha)-\frac{h}{q^{k}}\right)^{2} \leqslant c \frac{h k q^{h}}{q^{k}}
$$

where $c$ is some absolute constant.

Proof. On the one hand, we have

$\sum_{1}:=\sum_{\alpha \in A_{q}^{h}} F_{\beta}(\alpha)=\#\left\{\left(\gamma_{1}, \gamma_{2}\right): \alpha=\gamma_{1} \beta \gamma_{2}\right\}=\sum_{\ell=0}^{h-k} q^{\ell} q^{h-\ell-k}=q^{h-k}(h-k+1)$

while on the other hand

$$
\sum_{2}:=\sum_{\alpha \in A_{q}^{h}} F_{\beta}^{2}(\alpha)=\#\left\{\left(\gamma_{1}, \gamma_{2}, \gamma_{3}, \gamma_{4}\right): \alpha=\gamma_{1} \beta \gamma_{2}=\gamma_{3} \beta \gamma_{4}\right\}
$$

Now, write

$$
\sum_{2}=\sum_{2,0}+\sum_{2,1}+\sum_{2,2}
$$

where in $\sum_{2,0}$, we impose the condition $\lambda\left(\gamma_{1}\right)=\lambda\left(\gamma_{3}\right)$, in $\sum_{2,1}$, we impose the condition $\lambda\left(\gamma_{1}\right)>\lambda\left(\gamma_{3}\right)$, and finally in $\sum_{2,2}$, we are restricted to $\lambda\left(\gamma_{1}\right)<\lambda\left(\gamma_{3}\right)$. In $\sum_{2,0}$, we have $\gamma_{1}=\gamma_{3}$, so that $\sum_{2,0}=\sum_{1}$.

Let $\sum_{2,1,1}$ be the number of those $\gamma_{1}, \gamma_{3}$ for which $\lambda\left(\gamma_{3}\right) \leqslant \lambda\left(\gamma_{1}\right)+k$, and $\sum_{2,1,2}$ be the number of those $\gamma_{1}, \gamma_{3}$ for which $\lambda\left(\gamma_{3}\right)>\lambda\left(\gamma_{1}\right)+k$. Since $\gamma_{3}$ is a prefix of $\gamma_{1} \beta$, it follows that it has no more than $k$ distinct values for a fixed $\gamma_{1}$, and therefore that $\sum_{2,1,1} \leqslant k \sum_{1}$. Assume now that $\lambda\left(\gamma_{3}\right)>\lambda\left(\gamma_{1}\right)+k$. Thus

$$
\begin{array}{|c|c|c|c|c|}
\leftarrow \ell_{1} \rightarrow \leftarrow k \rightarrow & \multicolumn{1}{c}{\leftarrow \ell_{2} \rightarrow \quad \leftarrow \ell_{3} \longrightarrow} \\
\hline \gamma_{1} & \beta & \gamma_{2} & \\
\hline
\end{array}
$$

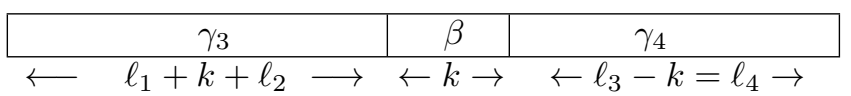

Let us fix the position of $\beta$ in (A) and in (B), that is the lengths $\ell_{1}$ and $\ell_{2}$. Then $\ell_{1}+\ell_{2}+\ell_{4}$ digits can be distributed freely, which yields $q^{\ell_{1}+\ell_{2}+\ell_{4}}=q^{h-2 k}$ integers. Hence the number of those nonnegative integers $\ell_{1}, \ell_{2}, \ell_{4}$ for which $\ell_{1}+\ell_{2}+\ell_{4}=h-2 k$ is therefore equal to

$$
\sum_{\ell_{4}=0}^{h-2 k}\left(h-2 k-\ell_{4}+1\right)=\sum_{\nu=1}^{h-2 k} \nu=\frac{(h-2 k)(h-2 k+1)}{2} .
$$

Thus

$$
\sum_{2,1,2}=\frac{(h-2 k)(h-2 k+1)}{2 q^{2 k}} q^{h}=\frac{h^{2} q^{h}}{2 q^{2 k}}+O\left(\frac{k h q^{h}}{q^{2 k}}\right)
$$


so that (3.16) can be written as

$$
\sum_{2}=\frac{h^{2} q^{h}}{q^{2 k}}+O\left(\frac{k h q^{h}}{q^{2 k}}\right),
$$

Therefore, using (3.15) and (3.17), inequality (3.14) follows, thus completing the proof of Lemma 6.

\section{Proof of Theorem 1}

Let $\wp^{*}=\bigcup_{j=0}^{q-1} \wp_{j}$ and define

$$
\omega_{\wp^{*}}(n):=\sum_{\substack{p \mid n \\ p \in \wp^{*}}} 1 .
$$

For each real number $u \geqslant 2$, let us set

$$
\rho_{u}:=T([u]+1) \ldots T([2 u]) .
$$

It is clear that

$$
\lambda\left(\rho_{u}\right)=u \sum_{\substack{p \leqslant 2 u \\ p \in \wp^{*}}} \frac{1}{p}+O(u)=q \delta u \log \log u+O(u) .
$$

Now let $k$ be a fixed positive integer and consider the word $\beta=i_{1} \ldots i_{k} \in A_{q}^{k}$. We now let $F_{\beta}(\alpha)$ stand for the number of occurrences of the word $\beta$ within the word $\alpha$, that is we set

$$
F_{\beta}(\alpha):=\#\left\{\left(\gamma_{1}, \gamma_{2}\right): \alpha=\gamma_{1} \beta \gamma_{2}, \gamma_{1}, \gamma_{2} \in A_{q}^{*}\right\} .
$$

We shall prove that

$$
\max _{\beta \in A_{q}^{k}}\left|F_{\beta}\left(\rho_{u}\right)-\frac{\lambda\left(\rho_{u}\right)}{q^{k}}\right| \leqslant \varepsilon(u) \lambda\left(\rho_{u}\right),
$$

where $\varepsilon(u)$ tends to 0 monotonically as $u \rightarrow \infty$.

Once we will have proven (4.2), Theorem 1 will follow. Indeed, let $\xi_{N}$ stand for the $q$-ary expansion of $\xi$ up to the $N$-th digit. Now, given $N$, let $u$ be a real number which satisfies the inequalities

$$
N_{1}:=\sum_{j \leqslant 2 u} \omega_{\wp^{*}}(j) \leqslant N<\sum_{j \leqslant 2 u+1} \omega_{\wp^{*}}(j) .
$$

Let us further set $\xi_{N_{1}}:=T(1) T(2) \ldots T([2 u])$. With this definition, we have that

$$
0 \leqslant \lambda\left(\xi_{N}\right)-\lambda\left(\xi_{N_{1}}\right)=O(\log N) .
$$


Now, given an arbitrary positive integer $\ell$ satisfying $2^{\ell}<u$, let us write

$$
\xi_{N_{1}}=\chi^{(\ell)} \rho_{u / 2^{\ell}} \rho_{u / 2^{\ell-1}} \ldots \rho_{u}
$$

where

$$
\rho_{v}:=T([v]+1) \ldots T([2 v])
$$

It follows that

$$
F_{\beta}\left(\xi_{N_{1}}\right)=F_{\beta}\left(\chi^{(\ell)}\right)+F_{\beta}\left(\rho_{u / 2^{\ell}}\right)+\cdots+F_{\beta}\left(\rho_{u}\right)+O(\ell+1) .
$$

Hence, using (4.2) and (4.3), we obtain that

$$
F_{\beta}\left(\xi_{N}\right)=F_{\beta}\left(\xi_{N_{1}}\right)+O(\log N)=\frac{\lambda\left(\xi_{N}\right)}{q^{k}}+O\left(\varepsilon\left(u / 2^{\ell}\right) N+\lambda\left(\chi^{(\ell)}\right)\right) .
$$

Now, choosing $\ell$ to be the unique integer satisfying $2^{\ell} \leqslant \sqrt{u}<2^{\ell+1}$ and using the fact that $\lambda\left(\chi^{(\ell)}\right) / N \rightarrow 0$ as $N \rightarrow \infty$, we then obtain from (4.4) that

$$
\frac{F_{\beta}\left(\xi_{N}\right)}{N} \rightarrow \frac{1}{q^{k}} \quad \text { as } N \rightarrow \infty
$$

thus proving that $\xi$ is a $q$-normal number.

Thus, it remains to prove (4.2). Doing so, we will make repetitive use of (4.1). First we set $w_{u}=\log \log \log u$ and $Z_{u}=\exp \left\{(\log u)^{1-\varepsilon_{u}}\right\}$, where $\varepsilon_{u} \rightarrow 0$ as $u \rightarrow \infty$, and write each integer $n \geqslant 2$ as

$$
n=\prod_{\substack{p^{a} \| n \\ p \leqslant w_{u}}} p^{a} \cdot \prod_{\substack{p^{a} \| n \\ w_{u}<p \leqslant Z_{u}}} p^{a} \cdot \prod_{\substack{p^{a} \| n \\ p>Z_{u}}} p^{a}=A(n) \cdot B(n) \cdot C(n),
$$

say. Since

$$
\sum_{u \leqslant n \leqslant 2 u} \omega(A(n))+\sum_{u \leqslant n \leqslant 2 u} \omega(C(n))=o(u \log \log u) \quad(u \rightarrow \infty),
$$

it follows that

$$
F_{\beta}\left(\rho_{u}\right)=\sum_{u \leqslant n \leqslant 2 u} F_{\beta}(T(B(n)))+o(u \log \log u) \quad(u \rightarrow \infty) .
$$

Let $\mathcal{M}_{u}$ be the set of those positive integers $m$ for which there exists at least one integer $n \in[u, 2 u]$ such that $B(n)=m$, in which case we let

$$
D(m)=\#\{n \in[u, 2 u]: B(n)=m\} .
$$

Then, from (4.6), we have

$$
F_{\beta}\left(\rho_{u}\right)=\sum_{m \in \mathcal{M}_{u}} F_{\beta}(T(m)) D(m)+o(u \log \log u) \quad(u \rightarrow \infty) .
$$


Further define $\mathcal{M}_{u}^{(1)}$ as the set of those $m \in \mathcal{M}_{u}$ for which at least one of the following conditions holds:

(1) $m$ is not squarefree,

(2) $m \geqslant Z_{u}^{K_{u}}, K_{u}=(\log u)^{\varepsilon_{u} / 2}$,

(3) there exist $p_{1} \mid m$ and $p_{2} \mid m$ such that $p_{1}<p_{2}<2 p_{1}$,

(4) $|\omega(m)-\log \log u|>(\log \log u)^{3 / 4}$.

Let $\mathcal{M}_{u}^{(0)}=\mathcal{M}_{u} \backslash \mathcal{M}_{u}^{(1)}$. Observing that $F_{\beta}(T(m)) \leqslant \omega(m)$, we easily obtain that

$$
\sum_{m \in \mathcal{M}_{u}^{(1)}} F_{\beta}(T(m)) D(m)=o(u \log \log u) \quad(u \rightarrow \infty) .
$$

By a standard sieve argument, we easily get that, as $u \rightarrow \infty$,

$$
D(m)=(1+o(1)) \frac{u}{m} \prod_{w_{u} \leqslant p \leqslant Z_{u}}\left(1-\frac{1}{p}\right)=(1+o(1)) \frac{u}{m} \frac{\log w_{u}}{\log Z_{u}} \quad\left(m \in \mathcal{M}_{u}^{(0)}\right) .
$$

Thus, using (4.8) and (4.9) in (4.7), we obtain

$$
F_{\beta}\left(\rho_{u}\right)=(1+o(1)) u \frac{\log w_{u}}{\log Z_{u}} \sum_{m \in \mathcal{M}_{u}^{(0)}} \frac{F_{\beta}(T(m))}{m}+o(u \log \log u) \quad(u \rightarrow \infty) .
$$

Hence, it remains to prove that, given arbitrary words $\beta_{1}$ and $\beta_{2}$ belonging to $A_{q}^{k}$,

$$
\sum_{m \in \mathcal{M}_{u}^{(0)}} \frac{F_{\beta_{1}}(T(m))}{m}=(1+o(1)) \sum_{m \in \mathcal{M}_{u}^{(0)}} \frac{F_{\beta_{2}}(T(m))}{m} \quad(u \rightarrow \infty) .
$$

We shall now use a technique we have already used to prove Theorem 1 of our 1995 paper [1]. We define the sequence $t_{0}<t_{1}<\cdots$ as follows:

$$
t_{0}=w_{u}, \quad t_{j+1}=t_{j}+\frac{t_{j}}{\left(\log t_{j}\right)^{5}} \quad \text { for } j=0,1, \ldots
$$

Let $r$ be defined implicitly by $t_{r} \leqslant Z_{u}<t_{r+1}$ and set $I_{j}=\left[t_{j}, t_{j+1}\right)$ for each integer $j \geqslant 0$.

Let $h$ be fixed, $|h-\log \log u| \leqslant(\log \log u)^{3 / 4}, 0 \leqslant j_{1}<j_{2}<\cdots<j_{h} \leqslant r-1$ with $j_{\ell+1} \geqslant 2 j_{\ell}$. Further define $\mathcal{M}_{u}^{(0)}\left(j_{1}, \ldots, j_{h}\right)$ as the set of those $m=\pi_{1} \pi_{2} \cdots \pi_{h}$ for which $\pi_{j} \in I_{\ell_{j}}$ for $j=1, \ldots, h$.

Observe that any $m \in \mathcal{M}_{u}^{(0)}\left(j_{1}, \ldots, j_{h}\right)$ satisfies

$$
\ell_{j_{1}+1} \cdot \ell_{j_{2}+1} \cdots \ell_{j_{h}+1} \geqslant m \geqslant \ell_{j_{1}} \cdot \ell_{j_{2}} \cdots \ell_{j_{h}}
$$


and that

$$
\begin{aligned}
1 & \leqslant \frac{\ell_{j_{1}+1} \cdot \ell_{j_{2}+1} \cdots \ell_{j_{h}+1}}{\ell_{j_{1}} \cdot \ell_{j_{2}} \cdots \ell_{j_{h}}} \leqslant \prod_{j=1}^{h}\left(1+\frac{1}{\left(\log \ell_{j}\right)^{5}}\right) \\
& \leqslant \exp \left\{\sum_{j=1}^{h} \frac{1}{\left(\log \ell_{j}\right)^{5}}\right\} \leqslant \exp \left\{\sum_{j=0}^{h-1} \frac{1}{\left(\log w_{u}+j \log 2\right)^{5}}\right\} \\
& =1+o(1) \quad(u \rightarrow \infty) .
\end{aligned}
$$

This means that instead of proving (4.10), we only need to prove

$$
\sum_{m \in \mathcal{M}_{u}^{(0)}\left(\ell_{j_{1}}, \ldots, \ell_{j_{h}}\right)} \frac{F_{\beta_{1}}(T(m))}{m}=(1+o(1)) \sum_{m \in \mathcal{M}_{u}^{(0)}\left(\ell_{j_{1}}, \ldots, \ell_{j_{h}}\right)} \frac{F_{\beta_{2}}(T(m))}{m} \quad(u \rightarrow \infty) .
$$

Now let $\mathcal{M}_{u}^{(0)}\left(\ell_{j_{1}}, \ldots, \ell_{j_{h}} \mid \wp_{\nu_{1}}, \ldots, \wp_{\nu_{h}}\right)$ be the set of those $m=\pi_{1} \pi_{2} \cdots \pi_{h} \in$ $\mathcal{M}_{u}^{(0)}\left(\ell_{j_{1}}, \ldots, \ell_{j_{h}}\right)$ for which $\pi_{\ell} \in \wp_{\nu_{\ell}}$.

Then, repeating the computation done in [1], we obtain that

$$
\frac{\# \mathcal{M}_{u}^{(0)}\left(\ell_{j_{1}}, \ldots, \ell_{j_{h}} \mid \wp_{\nu_{1}}, \ldots, \wp_{\nu_{h}}\right)}{\# \mathcal{M}_{u}^{(0)}\left(\ell_{j_{1}}, \ldots, \ell_{j_{h}}\right)}=(1+o(1)) \tau\left(\nu_{1}\right) \cdots \tau\left(\nu_{h}\right) \quad(u \rightarrow \infty),
$$

where $\tau(\nu)=\delta$ if $\nu \in\{0,1, \ldots, q-1\}$ and $\tau(q)=1-q \delta$. Assume that among $\nu_{1}, \ldots, \nu_{h}$, the value $q$ occurs $t_{1}$ times. Then, on the right hand side of (4.12), $\tau\left(\nu_{1}\right) \cdots \tau\left(\nu_{h}\right)=(1-q \delta)^{t_{1}} \cdot \delta^{h-t_{1}}$, which does depend only on $t_{1}$. It is clear that $F_{\beta}(T(m))$ is constant in every $\mathcal{M}_{u}^{(0)}\left(\ell_{j_{1}}, \ldots, \ell_{j_{h}} \mid \wp_{\nu_{1}}, \ldots, \wp_{\nu_{h}}\right)$. So, let $e_{1}<\cdots<$ $e_{t_{1}} \leqslant h$ be arbitrary integers and consider those $\wp_{\nu_{1}}, \ldots, \wp_{\nu_{h}}$ for which $\nu_{e_{j}}=q$ for $j=1, \ldots, t_{1}$ and $\nu_{\ell} \neq q$ if $\ell \neq e_{j}$. Let $v_{0}<v_{1}<\cdots<v_{h-t_{1}-1}$ be the sequence of integers defined by

$$
\left\{v_{0}, \ldots, v_{h-t_{1}-1}\right\}=\{1, \ldots, h\} \backslash\left\{e_{1}, \ldots, e_{t_{1}}\right\} .
$$

Moreover, let $\nu_{v_{j}} \in\{0,1, \ldots, q-1\}$ for $j=0,1, \ldots, h-t_{1}-1$ be arbitrary digits. If $m \in \mathcal{M}_{u}^{(0)}\left(\ell_{j_{1}}, \ldots, \ell_{j_{h}} \mid \wp_{\nu_{1}}, \ldots, \wp_{\nu_{h}}\right)$, then

$$
F_{\beta}(T(m))=F_{\beta}\left(\nu_{v_{0}} \nu_{v_{1}} \ldots \nu_{v_{h-t_{1}-1}}\right) .
$$

Now, one can easily show that the number of those $n \in[u, 2 u]$ for which $h-t_{1} \leqslant k^{2}$ is $o(u)$. Hence, we may assume that $h-t_{1}>k^{2}$. Then, in light of (4.12), (4.13) and Lemma 6, we easily obtain (4.11) and thereby (4.2) and (4.5), thus completing the proof of Theorem 1 .

\section{Proof of Theorem 2}

To prove Theorem 2, we use the notations of Theorem 1 and essentially the same kind of technique. 
Let $u \geqslant 3$ and set

$$
\kappa_{u}:=T\left(p_{1}-1\right) \ldots T\left(p_{k}-1\right),
$$

where $u \leqslant p_{1}<\cdots<p_{k}<2 u$ are all the primes included in the interval $[u, 2 u)$.

Now,

$$
\lambda\left(\kappa_{u}\right)=\sum_{u \leqslant p_{\ell}<2 u} \omega_{\wp^{*}}\left(p_{\ell}-1\right)=q \delta \cdot \frac{u}{\log u} \cdot \log \log u+o\left(\frac{u \log \log u}{\log u}\right),
$$

an estimate that follows essentially from the fact that $p-1$ has no more than 3 prime divisors $\pi>u^{1 / 3}$ if $u \leqslant p<2 u$. Since equation (5.1) provides the exact size of $\lambda\left(\kappa_{u}\right)$, it will be used repetitively below.

Then, choose the intervals $I_{j}$ as we did in the proof of Theorem 1 and let us estimate

$$
W(u):=\sum_{\substack{u \leqslant p_{\ell} \leqslant 2 u \\ \pi_{1}\left|p_{\ell}-1, \pi_{2}\right| p_{\ell}-1 \\ \pi_{1}, \pi_{2} \in I_{j} \text { for some } j}} \omega\left(p_{\ell}-1\right) .
$$

We have

$$
W(u)=\sum_{w_{u}<\pi_{1}<\pi_{2}<2 \pi_{1}<Z_{u}} \sum_{\substack{p \in(u, 2 u) \\ p-1 \equiv 0}} \omega(p-1) .
$$

Since $\pi_{1} \pi_{2} \mid p-1$, we have from (5.2) that

$$
W(u) \leqslant \sum_{w_{u}<\pi_{1}<\pi_{2}<2 \pi_{1}<Z_{u}} \sum_{\pi_{0}<u^{1 / 4}} \pi\left(2 u ; \pi_{0} \pi_{1} \pi_{2}, 1\right)+\pi\left(2 u ; \pi_{1} \pi_{2}, 1\right) .
$$

Then, using the Brun-Titchmarsh inequality (Lemma 2), it is clear that the right hand side of (5.3) is less than

$$
\begin{aligned}
c \frac{u}{\log u} \sum_{\pi_{0}<u^{1 / 4}} \frac{1}{\pi_{0}} \sum_{w_{u}<\pi_{1}<\pi_{2}<2 \pi_{1}<Z_{u}} \frac{1}{\left(\pi_{1}-1\right)\left(\pi_{2}-1\right)} \\
\quad+c \frac{u}{\log u} \sum_{w_{u}<\pi_{1}<\pi_{2}<2 \pi_{1}<Z_{u}} \frac{1}{\left(\pi_{1}-1\right)\left(\pi_{2}-1\right)} .
\end{aligned}
$$

On the other hand, one can easily establish that

$$
\sum_{\pi_{1}<\pi_{2}<2 \pi_{1}} \frac{1}{\left(\pi_{1}-1\right)\left(\pi_{2}-1\right)} \leqslant c \sum_{p} \frac{1}{(p-1) \log p}<\infty,
$$

while it is clear that

$$
\sum_{\pi_{0}<u^{1 / 4}} \frac{1}{\pi_{0}} \ll \log \log u
$$

Using estimates (5.5) and (5.6) in (5.4), it follows that (5.3) can be replaced by

$$
W(u)=o\left(\frac{u \log \log u}{\log u}\right) .
$$


Now, let $\beta=i_{1} \ldots i_{k} \in A_{q}^{k}$. We will now estimate $F_{\beta}\left(\kappa_{u}\right)$. We have

$$
F_{\beta}\left(\kappa_{u}\right)=\sum_{j=1}^{k} F_{\beta}\left(T\left(p_{j}-1\right)\right)+O(\pi([u, 2 u])) .
$$

Since

$$
\sum_{u \leqslant p \leqslant 2 u} \sum_{\substack{\pi_{0} \mid p-1 \\ \pi_{0} \notin\left(w_{u}, z_{u}\right)}} 1=o(\operatorname{li}(u) \log \log u) \quad(u \rightarrow \infty),
$$

relation (5.8) becomes

$$
F_{\beta}\left(\kappa_{u}\right)=\sum_{j=1}^{k} F_{\beta}\left(T\left(A\left(p_{j}-1\right) B\left(p_{j}-1\right)\right)\right)+o(\operatorname{li}(u) \log \log u) \quad(u \rightarrow \infty) .
$$

Let $\mathcal{A}([u, 2 u] \mid m)=\mathcal{A}(2 u \mid m)-\mathcal{A}(u \mid m)$. Using the notation of Lemma 5 , it follows that $A\left(p_{j}-1\right) B\left(p_{j}-1\right)=m$ holds for $\mathcal{A}([u, 2 u] \mid m)$ numbers, so that

$$
\sum_{\substack{m \leqslant Z_{u}^{K u} \\ P(m) \leqslant Z_{u}}}\left|\mathcal{A}([u, 2 u] \mid m)-\frac{\nu(Q) \kappa(m)}{m}(\operatorname{li}(2 u)-\operatorname{li}(u))\right| \ll \frac{u}{\log ^{C} u},
$$

where $C$ is an arbitrary positive number. Using this in (5.9), it follows that, with $\nu(Q)=\prod_{2<p \leqslant Z_{u}}\left(1-\frac{1}{p-1}\right)$ and with the strongly multiplicative function $\kappa$ defined in (3.1),

$$
F_{\beta}\left(\kappa_{u}\right)=\sum_{\substack{m \leqslant Z_{u}^{K u} \\ P(m) \leqslant Z_{u}}}^{*} \frac{\nu(Q) \kappa(m)}{m}(\operatorname{li}(2 u)-\operatorname{li}(u)) F_{\beta}(T(m))+o(\operatorname{li}(u) \log \log u),
$$

where the star $(*)$ on the sum indicates that, in light of (5.7), we omitted those $m$ 's for which there exist two primes $\pi_{1}$ and $\pi_{2}$ such that $\pi_{1} \pi_{2} \mid m, w_{u}<\pi_{1}<\pi_{2} \leqslant Z_{u}$ with $\pi_{1}, \pi_{2} \in I_{j}$ for some $j$. Note that in this same sum, we dropped those integers $m>Z_{u}^{K_{u}}$ since

$$
\sum_{\substack{m>Z_{u}^{K_{u}} \\ P(m) \leqslant Z_{u}}} \mathcal{A}([u, 2 u] \mid m) \omega(m)=o\left(\frac{u}{\log u} \log \log u\right) .
$$

Let us write

$$
m=\prod_{\substack{p^{a} \| m \\ p \leqslant w_{u}}} p^{a} \cdot \prod_{\substack{p^{a} \| m \\ w_{u}<p \leqslant z_{u}}} p^{a}=A m_{1},
$$

say. Since $\omega(A) \leqslant w_{u}=O(\log \log \log u)$, it follows that estimate (5.10) becomes

$$
F_{\beta}\left(\kappa_{u}\right)=\nu(Q)(\operatorname{li}(2 u)-\operatorname{li}(u)) \sum_{A}^{+} \frac{\kappa(A)}{A} \sum_{m_{1}}^{*} \frac{\kappa\left(m_{1}\right)}{m_{1}} F_{\beta}\left(T\left(m_{1}\right)\right)+o(\operatorname{li}(u) \log \log u),
$$


where the $(+)$ sign on the sum indicates that we dropped those integers $A$ which are large, namely those for which $A \geqslant w_{u}^{w_{u}}$, say.

Now, since

$$
\begin{aligned}
1 \leqslant \kappa\left(m_{1}\right) & =\prod_{p \mid m_{1}}\left(1+\frac{1}{p}\right) \prod_{p \mid m_{1}} \frac{p^{2}-p}{p^{2}-p-2} \\
& =\exp \left(\sum_{p \mid m_{1}} \log \left(1+\frac{2}{p^{2}-p-2}\right)\right) \prod_{p \mid m_{1}}\left(1+\frac{1}{p}\right) \\
& \leqslant \exp \left(3 \sum_{p>w_{u}} \frac{1}{p^{2}}\right) \prod_{p \mid m_{1}}\left(1+\frac{1}{p}\right) \\
& \leqslant\left(1+\frac{1}{w_{u}}\right) \sum_{d \mid m_{1}} \frac{|\mu(d)|}{d}
\end{aligned}
$$

we obtain that

$$
0 \leqslant \kappa\left(m_{1}\right)-1 \leqslant \sum_{\substack{d \mid m_{1} \\ d>1}} \frac{|\mu(d)|}{d}+\frac{1}{w_{u}} \sum_{d \mid m_{1}} \frac{|\mu(d)|}{d} .
$$

Hence, using this last estimate in (5.11), we obtain

$$
F_{\beta}\left(\kappa_{u}\right)=\nu(Q) \frac{u}{\log u} \sum_{A} \frac{\kappa(A)}{A} \sum_{m_{1}}^{*} \frac{1}{m_{1}} F_{\beta}\left(T\left(m_{1}\right)\right)+o(\operatorname{li}(u) \log \log u) .
$$

Proceeding as we did in the proof of Theorem 1, we then obtain that

$$
\sum_{m_{1}}^{*} \frac{1}{m_{1}} F_{\beta_{1}}\left(T\left(m_{1}\right)\right)=(1+o(1)) \sum_{m_{1}}^{*} \frac{1}{m_{1}} F_{\beta_{2}}\left(T\left(m_{1}\right)\right) \quad(u \rightarrow \infty),
$$

and therefore that

$$
F_{\beta_{1}}\left(\kappa_{u}\right)=F_{\beta_{2}}\left(\kappa_{u}\right)+o\left(\frac{u}{\log u} \log \log u\right) \quad(u \rightarrow \infty),
$$

for every words $\beta_{1}$ and $\beta_{2}$ belonging to $A_{q}^{k}$. This observation concludes the proof of Theorem 2.

\section{The proof of Theorem 3}

We first introduce the multiplicative function $\rho$ defined on prime powers by

$$
\rho\left(p^{a}\right)= \begin{cases}1 & \text { if } p=2 \text { and } a=1, \\ 0 & \text { if } p=2 \text { and } a \geqslant 2, \\ 2 & \text { if } p \equiv 1(\bmod 4), \\ 0 & \text { if } p \equiv 3(\bmod 4)\end{cases}
$$


First observe that, given an integer $D>1$, the congruence $n^{2}+1 \equiv 0(\bmod D)$ has $\rho(D)$ distinct solutions.

As in the proof of Theorem 1, let $Z_{u}=\exp \left\{(\log u)^{1-\varepsilon_{u}}\right\}$ and, for each integer $n \geqslant 2$, set

$$
E\left(n \mid Z_{u}\right)=\prod_{\substack{p^{a} \| n \\ p \leqslant Z_{u}}} p^{a} .
$$

Further define

$$
\mathcal{A}([u, 2 u] \mid m):=\#\left\{n \in[u, 2 u]: E\left(n^{2}+1 \mid Z_{u}\right)=m\right\}
$$

and let $K_{u}$ be a function tending to infinity with $u$ and that we will determine later.

Our first goal will be to find an upper bound for

$$
S:=\sum_{\substack{m>Z_{u}^{K u} \\ P(m) \leqslant z_{u}}} \mathcal{A}([u, 2 u] \mid m) \omega(m) .
$$

Letting $\omega_{Y}(n):=\sum_{p \mid n, p \leqslant Y} 1$, we have that

$$
\begin{aligned}
\sum_{n \in[u, 2 u]} \omega_{Z_{u}}\left(n^{2}+1\right)^{2} & \ll u \sum_{p_{1}, p_{2} \leqslant Z_{u}} \frac{\rho\left(p_{1}\right)}{p_{1}} \frac{\rho\left(p_{2}\right)}{p_{2}}+u \sum_{p \leqslant Z_{u}} \frac{\rho(p)}{p} \\
& \ll u\left(\log \log Z_{u}\right)^{2} \ll u(\log \log u)^{2} .
\end{aligned}
$$

Hence, it follows from this that, setting

$$
S_{1}:=\sum_{\substack{m>Z_{u}^{K u} \\ P(m) \leqslant Z_{u}}} \mathcal{A}([u, 2 u] \mid m),
$$

we have, letting $E(u)$ be a function which tends to infinity with $u$ and that we will determine later,

$$
\begin{aligned}
S & =\sum_{\substack{m>Z_{u}^{K} u \\
P(m) \leqslant Z_{u} \\
\omega_{Z_{u}}(m)<E(u) \log \log u}} \mathcal{A}([u, 2 u] \mid m) \omega(m)+\sum_{\substack{m>Z_{u}^{K} K_{u} \\
P(m) \leqslant Z_{u} \\
\omega_{Z_{u}}(m) \geqslant E(u) \log \log u}} \mathcal{A}([u, 2 u] \mid m) \omega(m) \\
& <E(u)(\log \log u) S_{1}+\sum_{\substack{n \in[u, 2 u] \\
\omega_{Z_{u}}\left(n^{2}+1\right) \geqslant E(u) \log \log u}} \omega_{Z_{u}}\left(n^{2}+1\right) \\
& \leqslant E(u)(\log \log u) S_{1}+\frac{1}{E(u) \log \log u} \sum_{n \in[u, 2 u]} \omega_{Z_{u}}\left(n^{2}+1\right)^{2} \\
& \ll E(u)(\log \log u) S_{1}+\frac{1}{E(u)} u \log \log u .
\end{aligned}
$$


Let us now bound $S_{1}$. Clearly, if $n \in[u, 2 u]$ is counted on the right hand side of (6.1), then the corresponding integer $m$ for which $E\left(n^{2}+1 \mid Z_{u}\right)=m>Z_{u}^{K_{u}}$ has a divisor $m_{1}$ satisfying $Z_{u}^{K_{u}-1} \leqslant m_{1} \leqslant Z_{u}^{K_{u}}$.

Now consider the subintervals $J_{\ell}:=\left[2^{\ell} Z_{u}^{K_{u}-1}, 2^{\ell+1} Z_{u}^{K_{u}-1}\right]$ with $\ell=0,1, \ldots, \ell_{0}$, where $\ell_{0}$ is the unique positive integer such that $2^{\ell_{0}-1} \leqslant Z_{u}<2^{\ell_{0}}$, so that

$$
\left[Z_{u}^{K_{u}-1}, Z_{u}^{K_{u}}\right] \subset \bigcup_{\ell=0}^{\ell_{0}} J_{\ell}
$$

Now, for a given integer $n \in[u, 2 u]$, if the corresponding integers $m$ and $m_{1}$ mentioned above are such that $m_{1}$ is the minimal divisor of $m$ and thus of $n^{2}+1$, with $m_{1} \in J_{\ell}$ for some $\ell \geqslant 0$, then $p\left(n^{2}+1\right)>2^{\ell}$. It follows from this that

$$
S_{1} \leqslant \sum_{\ell=0}^{\ell_{0}} T_{\ell}
$$

where

$T_{\ell} \leqslant \sum_{\substack{m_{1} \in J_{\ell} \\ P\left(m_{1}\right) \leqslant Z_{u} \\ p\left(m_{1}\right)>2^{\ell}}} \#\left\{n \in[u, 2 u]: m_{1} \mid n^{2}+1,\left(n^{2}+1, \pi_{0}\right)=1\right.$ for all primes $\left.\pi_{0}<2^{\ell}\right\}$.

Now, using the Selberg Sieve, it follows that

$$
\begin{aligned}
T_{\ell} & \leqslant u \sum_{\substack{m_{1} \in J_{\ell} \\
P\left(m_{1}\right) \leqslant Z_{u} \\
p\left(m_{1}\right)>2^{\ell}}} \frac{\rho\left(m_{1}\right)}{m_{1}} \prod_{\pi_{0}<2^{\ell}}\left(1-\frac{\rho\left(\pi_{0}\right)}{\pi_{0}}\right) \ll \frac{u}{\ell} \sum_{\substack{m_{1} \in J_{\ell} \\
P\left(m_{1}\right) \leqslant Z_{u} \\
p\left(m_{1}\right)>2^{\ell}}} \frac{\rho\left(m_{1}\right)}{m_{1}} \\
& \leqslant \frac{u}{\ell} \frac{1}{2^{\ell} Z_{u}^{K_{u}-1}} \sum_{\substack{m_{1} \in J_{\ell} \\
P\left(m_{1} \leqslant \leqslant Z_{u} \\
p\left(m_{1}\right)>2^{\ell}\right.}} \rho\left(m_{1}\right)=\frac{u}{\ell} \frac{1}{2^{\ell} Z_{u}^{K_{u}-1}} H_{\ell},
\end{aligned}
$$

say.

Again, using a sieving technique, we get that

$$
H_{\ell} \ll \prod_{p<2^{\ell}}\left(1-\frac{\rho(p)}{p}\right) \prod_{Z_{u}<p<Z_{u}^{K u}}\left(1-\frac{\rho(p)}{p}\right) \sum_{a \in J_{\ell}} \rho(a) \ll \frac{1}{\ell K_{u}} 2^{\ell} Z_{u}^{K_{u}-1} .
$$

Using (6.5) in (6.4), we obtain that

$$
T_{\ell} \ll \frac{u}{\ell^{2} K_{u}} .
$$

Substituting this back in (6.3), we obtain that

$$
S_{1} \ll \frac{u}{K_{u}} .
$$


Using this in (6.2) and choosing $E(u)$ and $K_{u}$ in such a manner that $E(u) / K_{u} \rightarrow 0$ as $u \rightarrow \infty$, we finally obtain that

$$
S=o(u \log \log u) \quad(u \rightarrow \infty) .
$$

Now assume that $m \leqslant Z_{u}^{K_{u}}$. Then, in light of (6.6) and using Lemma 1, we obtain that

$$
\mathcal{A}([u, 2 u] \mid m)=(1+o(1)) u \frac{\rho(m)}{m} \prod_{\pi_{0}<Z_{u}}\left(1-\frac{\rho\left(\pi_{0}\right)}{\pi_{0}}\right) \quad(u \rightarrow \infty) .
$$

The rest of the proof of Theorem 3 then runs along the same lines as those of Theorems 1 and 2, in particular by using our 1995 results (see [1]).

Indeed, let

$$
\tau_{u}:=T(f([u]+1)) \ldots T(f([2 u])) .
$$

By using (6.7) and our earlier estimates, we obtain that, in light of (6.6), as $u \rightarrow \infty$,

$$
F_{\beta}\left(\tau_{u}\right)=(1+o(1)) u \prod_{\pi_{0}<Z_{u}}\left(1-\frac{\rho\left(\pi_{0}\right)}{\pi_{0}}\right) \sum_{\substack{m \leqslant Z_{u}^{K} \\ P(m) \leqslant Z_{u}}} \frac{\rho(m)}{m} F_{\beta}(T(m))+o(u \log \log u) .
$$

Let $w_{u}=\log \log \log u$ and write $m$ in the form $m=A m_{1}$, where $P(A) \leqslant w_{u}$, $p\left(m_{1}\right)>w_{u}$. As in our 1995 paper [1], we let $\ell_{j}$, for $j=0,1, \ldots$, be the sequence defined by

$$
\ell_{0}=w_{u} \quad \text { and } \quad \ell_{j+1}=\ell_{j}+\frac{\ell_{j}}{\left(\log \ell_{j}\right)^{5}}
$$

and set $I_{j}:=\left[\ell_{j}, \ell_{j+1}\right]$.

Now, on the right hand side of (6.8), drop those integers $m=A m_{1}$ for which one of the following five conditions holds:

1. $m_{1}$ is not squarefree,

2. $A>w_{u}^{w_{u}}$

3. there exist two prime divisors $q_{1}, q_{2}$ of $m_{1}$ for which $q_{1}<q_{2}<4 q_{1}$,

4. $\left|\omega\left(m_{1}\right)-\log \log u\right|>\frac{1}{2} \log \log u$,

5. $\rho(m)=0$.

It is easy to see that the whole contribution of the dropped elements located on the right hand side of $(6.8)$ is $o(u \log \log u)$.

Let us now consider all the remaining $m$ 's. Let $m_{1}=q_{1} q_{2} \cdots q_{h}$ and let $\ell_{i_{1}}, \ldots, \ell_{i_{h}}$ be such indices for which $q_{j} \in I_{\ell_{j}}(j=1, \ldots, h)$. Further let $\mathcal{K}\left(\ell_{i_{1}}, \ldots, \ell_{i_{h}}\right)$ be the set of those $m_{1}$ 's for which each factor $q_{j} \mid m_{1}$ belongs to $I_{\ell_{j}}$ for $j=1, \ldots, h$. We then have

$$
\min m_{1} \geqslant \prod_{j=1}^{h} \ell_{i_{j}} \quad \text { and } \quad \max m_{1} \leqslant\left(\prod_{j=1}^{h} \ell_{i_{j}}\right) \exp \left\{\sum_{j=1}^{h} \frac{c}{\left(\log \ell_{i_{j}}\right)^{5}}\right\} .
$$


But since $\ell_{i_{j}} \geqslant 4^{j-1} \ell_{0}$, it follows that

$$
\sum_{j=1}^{h} \frac{c}{\left(\log \ell_{i_{j}}\right)^{5}} \leqslant \sum_{j=1}^{h} \frac{c}{\left(\log \ell_{0}+(j-1) \log 4\right)^{5}} \leqslant \frac{c_{1}}{\left(\log \ell_{0}\right)^{5}},
$$

and therefore $m_{1} \in \mathcal{K}\left(\ell_{i_{1}}, \ldots, \ell_{i_{h}}\right)$ implies that

$$
\frac{\rho\left(m_{1}\right)}{m_{1}}=(1+o(1)) 2^{h} \prod_{j=1}^{h} \ell_{i_{j}} \quad(u \rightarrow \infty),
$$

implying that, for those $m_{1} \in \mathcal{K}\left(\ell_{i_{1}}, \ldots, \ell_{i_{h}}\right)$, we have that $\frac{\rho\left(m_{1}\right)}{m_{1}}$ tends to a constant (independent of $m_{1}$ ) as $u \rightarrow \infty$.

Now, the number of integers $m_{1} \in \mathcal{K}\left(\ell_{i_{1}}, \ldots, \ell_{i_{h}}\right)$ is equal to

$$
D:=\prod_{j=1}^{h} \pi\left(I_{\ell_{i_{j}}} \cap \widetilde{\wp}\right) \text {. }
$$

Let us set $\mathcal{R}_{0}=\widetilde{\wp} \backslash \bigcup_{j=0}^{q-1} \wp_{i}$. On the one hand, it is obvious that, in light of $(2.1)$,

$$
\begin{aligned}
\pi\left([u, v] \cap \mathcal{R}_{0}\right) & =\pi(u+v ; 4,1)-\pi(u ; 4,1)-\sum_{j=0}^{q-1} \pi\left([u, v] \cap \wp_{j}\right) \\
& =\left(\frac{1}{2}-q \delta\right) \pi([u, v])+O\left(\frac{u}{\log ^{c_{1}} u}\right)
\end{aligned}
$$

uniformly for $2 \leqslant v \leqslant u$.

We shall now subdivide the sequence $\left\{i_{1}, \ldots, i_{h}\right\}$ into two monotone subsequences $\left\{u_{1}, \ldots, u_{t}\right\}$ and $\left\{v_{1}, \ldots, v_{h-t}\right\}$ in the following way. Count those elements of $\mathcal{K}\left(\ell_{i_{1}}, \ldots, \ell_{i_{h}}\right)$ in which $q_{u_{j}} \in \mathcal{R}_{0}$ and $q_{v_{\nu}} \in \wp_{e_{\nu}}$ for $e_{\nu} \in\{0, \ldots, q-1\}$ $(\nu=1, \ldots, h-t)$, and denote this number by $\mathcal{D}\left(u_{1}, \ldots, u_{t} \mid e_{1}, \ldots, e_{h-t}\right)$. Then, in light of the definition of $D$ given in (6.9) and of estimate (6.10), it follows that, uniformly for $0 \leqslant t \leqslant h$,

$$
\frac{\mathcal{D}\left(u_{1}, \ldots, u_{t} \mid e_{1}, \ldots, e_{h-t}\right)}{D}=(1+o(1))\left(\frac{1}{2}-q \delta\right)^{t} \delta^{h-t} \quad(h \rightarrow \infty) .
$$

Hence, we obtain that for every class $\mathcal{K}\left(\ell_{i_{1}}, \ldots, \ell_{i_{h}}\right)$, we have that

$$
\begin{aligned}
\sum_{m \in \mathcal{K}\left(\ell_{i_{1}}, \ldots, \ell_{i_{h}}\right)} \frac{\rho\left(m_{1}\right)}{m_{1}} & F_{\beta_{1}}\left(T\left(m_{1}\right)\right) \\
& =(1+o(1)) \sum_{m \in \mathcal{K}\left(\ell_{i_{1}}, \ldots, \ell_{i_{h}}\right)} \frac{\rho\left(m_{1}\right)}{m_{1}} F_{\beta_{2}}\left(T\left(m_{1}\right)\right) \quad(h \rightarrow \infty),
\end{aligned}
$$


which in turn implies that

$$
F_{\beta_{1}}\left(\tau_{u}\right)=F_{\beta_{2}}\left(\tau_{u}\right)+o(u \log \log u) \quad(u \rightarrow \infty)
$$

for every words $\beta_{1}$ and $\beta_{2}$ belonging to $A_{q}^{k}$, which completes the proof of Theorem 3.

\section{References}

[1] J.M. De Koninck and I. Kátai, On the distribution of subsets of primes in the prime factorization of integers, Acta Arithmetica 72 (1995), no.2, 169-200.

[2] J.M. De Koninck and I. Kátai, Construction of normal numbers by classified prime divisors of integers, Functiones et Approximatio 45.2 (2011), 231-253.

[3] P.D.T.A. Elliott, Probabilistic Number Theory I, Mean Value Theorems, Springer-Verlag, Berlin, 1979.

[4] H.H. Halberstam and H.E. Richert, Sieve Methods, Academic Press, London, 1974.

[5] H. Iwaniec and E. Kowalski, Analytic Number Theory, AMS Colloquium Publications, Vol. 53, 2004.

Addresses: Jean-Marie De Koninck: Département de mathématiques et de statistique, Université Laval, Québec, Québec G1V 0A6, Canada;

Imre Kátai: Computer Algebra Department, Eötvös Loránd University, 1117 Budapest, Pázmány Péter Sétány I/C, Hungary.

E-mail: jmdk@mat.ulaval.ca, katai@compalg.inf.elte.hu

Received: 9 January 2012 